\title{
Testing for Structural Change of Predictive Regression Model to Threshold Predictive Regression Model*
}

\author{
Fukang Zhu ${ }^{a}$, Mengya Liu ${ }^{a}$, Shiqing Ling ${ }^{b *}$ and Zongwu $\mathrm{Cai}^{c}$ \\ ${ }^{a}$ School of Mathematics, Jilin University, Changchun, Jilin 130012, China \\ ${ }^{b}$ Department of Mathematics, Hong Kong University of Science and Technology \\ ${ }^{c}$ Department of Economics, University of Kansas, Lawrence, KS 66025, USA
}

\begin{abstract}
This paper investigates two test statistics for structural changes and thresholds in predictive regression models. The generalized likelihood ratio (GLR) test is proposed for the stationary predictor and the generalized F test is suggested for the unit root predictor. Under the null hypothesis of no structural change and threshold, it is shown that the GLR test statistic converges to a function of a centered Gaussian process, and the generalized $\mathrm{F}$ test statistic converges to a function of Brownian motions. A Bootstrap method is proposed to obtain the critical values of test statistics. Simulation studies and a real example are given to assess the performances of the tests.
\end{abstract}

Key Words: Bootstrap method, Generalized F-test; Generalized likelihood ratio test; Predictive regression, Structural change, Threshold model.

JEL Classification: C12, C22, C58, G12.

${ }^{*}$ Corresponding author: Shiqing Ling (E-mail: maling@ust.hk). 


\section{Introduction}

The question of whether asset returns are predictable or not is one of the most studied and contentious issues in financial economics. Predictors commonly considered for returns include various lagged financial variables, such as log dividend-price ratio, log earnings-price ratio, log book-tomarket ratio, dividend yield, term spread, term structure of interest rates, and default premia. Predictive regression (PR) is a conventional method to check whether some financial variables have the explanatory power on the stock return predictability. It is extensively utilized in studies of mutual fund performance, conditional capital asset pricing and optimal asset allocation; see, for example, the survey paper by Liao et al. (2018). The classical PR model takes the following simple form

$$
y_{t}=\psi_{0}+\psi_{1} x_{t-1}+u_{t}
$$

where $u_{t}$ is commonly assumed to be an independent and identically distributed (i.i.d.) innovation process with mean 0 and variance $\sigma^{2}>0$ and the predictor $x_{t}$ is modeled as

$$
x_{t}=\rho x_{t-1}+v_{t}
$$

where $v_{t}$ is a mean zero innovation process. For convenience, we set $x_{0}=0$. Inferences in PR models are complicated due to the joint interaction of the highly persistent nature $(|\rho|$ in $(1.2)$ can be either one or very close to one) of the commonly used predictors with endogeneity problems arising from the correlation of $u_{t}$ and $v_{t}$. There is growing literature aiming to develop valid and reliable inferences in such settings, see Campbell and Yogo (2006), Jansson and Moreira (2006), Cai and Wang (2014), Zhu et al. (2014a) and Breitung and Demetrescu (2015), Yang et al. (2020, 2021), among others. For more recent developments in this area, the reader is referred to the survey paper by Liao et al. (2018).

It is well documented that predictability may be time-varying and that the impact of predictors may be evolving over time. In a comprehensive study on the predictability of the equity premium, Welch and Goyal (2008) found significant instabilities in predictability as highlighted by others. The sensitivity analysis conducted in Kostakis et al. (2015) also highlighted significant variations in test conclusions which depend on whether one considers pre- or post-50s data. There are two common modeling tools to deal with parameter instability in PR models: structural change and threshold model. 
Testing for structural change has always been an important issue in econometrics because a myriad of political and economic factors can cause the relationships among economic variables to change over time. The great depression, oil price shocks, technical progress, and abrupt policy and regulations changes all are such examples. The earliest references go back to Chow (1960) and Quandt (1960). The Chow's test assumes that the time of structural change is known a priori, while Quandt's test takes the largest Chow test statistic over all possible times of the structural change. This type of test statistics needs a normalization and has a Darling-Erdös-type limit, see Horváth (1993) and Ling (2007). Another method is to restrict the change-point interval $(0,1)$ to a closed subinterval, see Andrews (1993) and Bai and Perron (1998). Recently, Pitarakis (2017) studied two cumulative squared residuals-based tests and Georgiev et al. (2018) considered the SupF and Cramér-von-Mises type statistics for a change point in PR models.

As pointed by Gonzalo and Pitarakis (2012), the predictive impact of a variable may alternate in sign/strength across different economically meaningful episodes (e.g., periods of rapid versus slow growth, periods of high versus low stock market valuation, periods of high versus low consumer confidence). Ignoring such phenomena by proceeding within a linear framework may mask the forecasting ability of a particular variable and the presence of interesting and economically meaningful dynamics. Actually, Gonzalo and Pitarakis (2012) investigated the threshold PR (TPR) model as follows:

$$
y_{t}=\psi_{0}+\psi_{1} x_{t-1}+\left(\phi_{0}+\phi_{1} x_{t-1}\right) I\left(q_{t-1} \leq r\right)+u_{t}
$$

where $q_{t}$ is a threshold (stationary) variable and $r$ is an unknown threshold parameter, and Gonzalo and Pitarakis $(2012,2017)$ applied the model in (1.3) to the prediction of stock returns with dividend yields and found the presence of regimes in which predictability kicks in solely during bad economic times. As a result, their analysis also illustrated the fact that the presence of regimes may make predictability appear as nonexistent when assessed within a linear model.

Besides the previous methods, some researchers believe that parameters should smoothly change, such as Cai et al. (2015) which developed a test against smooth parameter variation in the parameters of the PR model. Existing test statistics just for the change of parameters or the form of the model (i.e., threshold). In practice, the structure of a model may be changed in terms of both time horizon and states. For example, the threshold AR-ARCH models were used to fit 11 non-overlapping 2-year period Hong Kong Hang Seng index from 1970 to 1991, but Wong and Li 
(1997) found that 2 out of 11 periods should follow the AR-ARCH models. When the form of models is changed, the conventional likelihood ratio test for the standard change-point problem is not locally most powerful any more. Therefore, how to efficiently detect the change of the structural forms of the time series models is of great interest. Indeed, Berkes et al. (2011) and Zhu and Ling (2012) considered this kind of problem in autoregressive models and provided several supporting real examples.

In this paper, we investigate two test statistics for structural changes and thresholds in PR models. The generalized likelihood ratio (GLR) test is proposed for the stationary predictor and the generalized F (GF) test is suggested for the unit root predictor. Under the null hypothesis of no structural change and threshold, it is shown that the GLR test statistic converges to a function of a centered Gaussian process, and the GF test statistic converges to a function of Brownian motions. Finally, to make the proposed tests useful in practice, a Bootstrap method is proposed to obtain the critical values of test statistics.

This paper is organized as follows. Section 2 presents the test statistics and their limiting distributions. Section 3 discusses a Bootstrap method to obtain the critical values of test statistics. Simulation studies and a real example are given to assess the finite sample performances of the proposed tests in Sections 4 and 5, respectively. Section 6 concludes the paper. All detailed proofs are given in the appendix.

\section{Test Statistics and Main Results}

To set up our setting, let $\left\{\left(y_{t}, x_{t}, q_{t}\right), t=1, \ldots, n\right\}$ be a sequence of observations from the model

$$
y_{t}= \begin{cases}\psi_{0}+\psi_{1} x_{t-1}+u_{t}, & t=1, \ldots, k \\ \psi_{0}+\psi_{1} x_{t-1}+\left(\phi_{0}+\phi_{1} x_{t-1}\right) I\left(q_{t-1} \leq r\right)+u_{t}, & t=k+1, \ldots, n\end{cases}
$$

where $x_{t}$ is defined as in (1.2). We consider the null and alternative hypotheses:

$$
H_{0}: k=n \text { v.s. } \quad H_{1}: k=k^{*} \text { and } \phi \neq 0,
$$

where $0<k^{*}<n$ and $\phi=\left(\phi_{0}, \phi_{1}\right)^{\top}$. The model in (2.1) is a PR model under $H_{0}$ and it changes to a TPR model after the time $k^{*}$ under $H_{1}$. Under $H_{0}$, no change has occurred and no threshold effect exists, while under $H_{1}$, a change has occurred at time $k^{*}$ (if $k^{*}>0$ ) and a threshold effect exists. When $r=\infty,(2.2)$ is for testing the parameter change in the PR model, which was studied 
recently by Pitarakis (2017) and Georgiev et al. (2018). When $k=0,(2.2)$ is for testing the threshold in the TPR model investigated by Gonzalo and Pitarakis $(2012,2017)$. Therefore, under $H_{0}$, both $k^{*}$ and $r$ are absent, which is the main difficulty in our setting.

Let $Y=\left(y_{1}, \ldots, y_{n}\right)^{\top}, X_{t}=\left(1, x_{t-1}\right)^{\top}, X=\left(X_{1}, \ldots, X_{n}\right)^{\top}, u=\left(u_{1}, \ldots, u_{n}\right)^{\top}, \psi=\left(\psi_{0}, \psi_{1}\right)^{\top}$, and $\theta=\left(\psi^{\top}, \phi^{\top}\right)^{\top}$. Denote $X_{k r}=\operatorname{diag}\left\{I\left(q_{i-1} \leq r, i>k\right), i=1, \ldots, n\right\} X$ and $\widetilde{X}_{k r}=\left(X, X_{k r}\right)$. Here, $X$ and $\tilde{X}_{k r}$ are $n \times 2$ and $n \times 4$, respectively. Under $H_{0}$, clearly, the model in (2.1) becomes to $Y=X \psi+u$, while under $H_{1}$, it reduces to $Y=\tilde{X}_{k r} \theta+u$. Therefore, the least squares based estimates of $\sigma^{2}$ under $H_{0}$ and $H_{1}$ are given by

$$
\hat{\sigma}_{n}^{2}=\frac{1}{n}\left[Y^{\top} Y-\left(Y^{\top} X\right)\left(X^{\top} X\right)^{-1}\left(X^{\top} Y\right)\right] \equiv \frac{1}{n} \mathrm{RSS}_{0}
$$

and

$$
\hat{\sigma}_{n}^{2}(k, r)=\frac{1}{n}\left[Y^{\top} Y-\left(Y^{\top} \widetilde{X}_{k r}\right)\left(\widetilde{X}_{k r}^{\top} \widetilde{X}_{k r}\right)^{-1}\left(\widetilde{X}_{k r}^{\top} Y\right)\right] \equiv \frac{1}{n} \mathrm{RSS}_{1},
$$

respectively, where $\mathrm{RSS}_{0}$ and $\mathrm{RSS}_{1}$ are the residual sum of squares (RSS) under $H_{0}$ and $H_{1}$, respectively.

\subsection{Stationary Predictor}

This subsection is devoted to the case when the predictor $x_{t}$ is stationary. That is $|\rho|<1$, where $\rho$ is given in (1.2). Let the filtration $\mathcal{F}_{t}$ be the $\sigma$-field generated by $\left\{\left(u_{i}, v_{i}, q_{i}\right): 1 \leq i \leq t\right\}$ and $F(\cdot)$ be the distribution function of $q_{t}$. We impose the following assumptions.

Assumption 1 Assume that the process $\left\{q_{t}\right\}$ is a stationary $\alpha$-mixing process with geometric rate and $|\rho|<1$, where $\rho$ is given in (1.2).

Assumption $2 E\left(u_{t} \mid \mathcal{F}_{t-1}\right)=0$ and $0<E\left[\left\|X_{t} u_{t}\right\|^{2}\left(\ln \left\|X_{t} u_{t}\right\|\right)^{5}\right]<\infty$, where $X_{t}=\left(1, x_{t-1}\right)^{\top}$ and $\|\cdot\|$ denotes the Euclidean norm.

Assumption $3 F(\cdot)$ is continuous and strictly increasing and the corresponding density function is bounded away from zero and $\infty$ over each bounded set.

Assumption 1 implies that $\left\{y_{t}\right\}$ is a stationary $\alpha$-mixing process with geometric rate and Assumption 2 allows that $u_{t}$ is a martingale difference sequence (MDS), and it permits correlation 
between innovations $u_{t}$ and $v_{t}$. For fixed $k$ and $r$, the GLR-type statistic for testing (2.2) is given by

$$
\left.\operatorname{GLR}_{n}(k, r)=n\left(\ln \hat{\sigma}_{n}^{2}-\ln \hat{\sigma}_{n}^{2}(k, r)\right)\right)
$$

which is the exact likelihood ratio if the distribution of $u_{t}$ is $N\left(0, \sigma^{2}\right)$; see, for example, Berkes et al. (2011) and Zhu and Ling (2012) for details. Note that $\operatorname{GLR}_{n}(k, r)$ in (2.5) can be expressed as

$$
\operatorname{GLR}_{n}(k, r)=n \ln \left(\frac{\mathrm{RSS}_{0}-\mathrm{RSS}_{1}}{\mathrm{RSS}_{1}}+1\right) \approx n\left(\frac{\mathrm{RSS}_{0}-\mathrm{RSS}_{1}}{\mathrm{RSS}_{1}}\right),
$$

and by Taylor expansion, it can be approximated by the F-type test statistic, termed as generalized F-test (GF) in Cai and Tiwari (2000) and Cai et al. (2019); see (2.7) later. Therefore, the GLR test is asymptotically equivalent to the GF test. Since the exact values of $k$ and $r$ are unknown under $H_{0}$, it is natural to construct our test by using the maxima of $\operatorname{GLR}_{n}(k, r)$ on the ranges of $k$ and $r$. That is, our test statistic is defined as

$$
\mathrm{GLR}_{n}=\sup _{0 \leq k \leq k_{1}} \sup _{r_{1} \leq r \leq r_{2}} \operatorname{GLR}_{n}(k, r)
$$

where $-\infty<r_{1}<r_{2}<\infty, k_{1}=\left\lfloor n \pi_{1}\right\rfloor$ with $0<\pi_{1}<1$, and $\left\lfloor n \pi_{1}\right\rfloor$ denotes the integer part of $n \pi_{1}$.

Let $I_{t-1}^{*}=I\left(q_{t-1} \leq r\right)$. Then, $I_{t-1}^{*}=I\left(F\left(q_{t-1}\right) \leq F(r)\right) \equiv I\left(U_{t-1} \leq \lambda\right)$, where $\lambda \equiv F(r)$ and $U_{t}$ is the uniformly distributed random variable on $[0,1]$. Define $\lambda_{1} \equiv F\left(r_{1}\right)$ and $\lambda_{2} \equiv F\left(r_{2}\right)$. Then, $\lambda \in\left[\lambda_{1}, \lambda_{2}\right]$. The following theorem gives the limiting distribution of $\mathrm{GLR}_{n}$.

Theorem 1 If Assumptions 1-3 hold, then under $H_{0}$, it follows that

$$
\mathrm{GLR}_{n} \stackrel{d}{\longrightarrow} \sup _{0 \leq \pi \leq \pi_{1}} \sup _{\lambda_{1} \leq \lambda \leq \lambda_{2}} \xi_{\pi \lambda}^{\top} M_{\pi \lambda}^{-1} \xi_{\pi \lambda} \text { as } n \rightarrow \infty
$$

where $\stackrel{d}{\longrightarrow}$ denotes convergence in distribution, $M_{\pi \lambda}=\Sigma_{\pi \lambda}-\Sigma_{\pi \lambda} \Sigma^{-1} \Sigma_{\pi \lambda}, \Sigma=E\left(X_{t} X_{t}^{\top}\right), \Sigma_{\pi \lambda}=$ $(1-\pi) E\left(I_{t-1}^{*} X_{t} X_{t}^{\top}\right), \xi_{\pi \lambda}$ is a Gaussian process with mean zero and covariance kernel

$$
\operatorname{Cov}\left(\xi_{\pi \lambda}, \xi_{\pi^{*} \lambda^{*}}\right)=\Sigma_{\max \left(\pi, \pi^{*}\right), \min \left(\lambda, \lambda^{*}\right)}-\Sigma_{\pi \lambda} \Sigma^{-1} \Sigma_{\pi^{*} \lambda^{*}}
$$

If $x_{t}$ and $q_{t}$ are independent, then $\Sigma_{\pi \lambda}=a_{\pi \lambda} \Sigma$. Thus, $M_{\pi \lambda}=a_{\pi \lambda}\left(1-a_{\pi \lambda}\right) \Sigma$ and the covariance kernel of $\xi_{\pi \lambda}$ is $\left[a_{\max \left(\pi, \pi^{*}\right), \min \left(\lambda, \lambda^{*}\right)}-a_{\pi \lambda} a_{\pi^{*} \lambda^{*}}\right] \Sigma$. This is the following corollary.

Corollary 1 Under the assumption of Theorem 1, if $x_{t}$ and $q_{t}$ are independent, then

$$
\mathrm{GLR}_{n} \stackrel{d}{\longrightarrow} \sup _{0 \leq \pi \leq \pi_{1}} \sup _{\lambda_{1} \leq \lambda \leq \lambda_{2}} \frac{\xi_{\pi \lambda}^{* \top} \xi_{\pi \lambda}^{*}}{a_{\pi \lambda}\left(1-a_{\pi \lambda}\right)}
$$

as $n \rightarrow \infty$, where $a_{\pi \lambda}=(1-\pi) \lambda$ and $\xi_{\pi \lambda}^{*}$ is a Gaussian process with mean zero and covariance kernel $\operatorname{Cov}\left(\xi_{\pi \lambda}^{*}, \xi_{\pi^{*} \lambda^{*}}^{*}\right)=\left[a_{\max \left(\pi, \pi^{*}\right), \min \left(\lambda, \lambda^{*}\right)}-a_{\pi \lambda} a_{\pi^{*} \lambda^{*}}\right] I_{2}$. 


\subsection{Unit Root Predictor}

This subsection considers the test statistic for (2.2) when $x_{t}$ is unit-root (or integrated or $\rho=1$ ) and near-integrated (or local-to-unity or $\rho=\rho_{n}=1-c / n$ for some $c>0$ ) predictor. Let $w_{t}=\left(u_{t}, v_{t}\right)^{\top}$. We impose the following maintained conditions on the model.

Assumption $4 E\left(w_{t} \mid \mathcal{F}_{t-1}\right)=0, E\left(w_{t} w_{t}^{\top} \mid \mathcal{F}_{t-1}\right)>0, \operatorname{Cov}\left(u_{t}, v_{t}\right)=0, \sup _{t} E u_{t}^{4}<\infty$, and $\sup _{t} E v_{t}^{4}<\infty$

Assumption 5 The threshold variable $q_{t}$ is strictly stationary, ergodic, and strong mixing with mixing coefficients $\alpha_{m}$ satisfying $\sum_{m=1}^{\infty} \alpha_{m}^{1 / 2-1 / \kappa}<\infty$ for $\kappa>2$.

Again, $w_{t}$ in Assumption 4 is a MDS, and it permits correlation between innovations $u_{t}$ and $v_{t}$ so that it can be used to establish the weak convergence result of the empirical process $\sum_{t=1}^{\lfloor n s\rfloor} I_{t-1}^{*} u_{t}$ as in Caner and Hansen (2001). It can be replaced by the following two assumptions: (i) $\left\{u_{t}, \mathcal{F}_{t}\right\}$ is a stationary and ergodic MDS with $E\left(u_{t}^{2} \mid \mathcal{F}_{t-1}\right)=\sigma^{2}, E\left(u_{t}\right)=0$, and $E\left(u_{t}^{4}\right)<\infty$. (ii) $\sum_{t=1}^{\lfloor n s\rfloor} w_{t} / \sqrt{n} \Longrightarrow\left(B_{u}(s), B_{v}(s)\right)^{\top}$ as $n \rightarrow \infty$, where $\left(B_{u}(s), B_{v}(s)\right)$ is a vector of Brownian motions with a positive definite long-run covariance matrix and “ $\Longrightarrow$ " represents weak convergence. Similar assumptions were made by Chen (2015) when studying the robust estimation and inference of threshold models with integrated regressors. Assumption 5 is very conventional in the literature of threshold models. As pointed out by Gonzalo and Pitarakis (2012), Assumption 4 implies that a functional central limit theorem holds for the joint process $w_{t}$, i.e., $\sum_{t=1}^{\lfloor n s\rfloor} w_{t} / \sqrt{n} \Longrightarrow\left(B_{u}(s), B_{v}(s)\right)^{\top}$, with the long-run variance of the bivariate Brownian motion being given by $\sum_{k=-\infty}^{\infty} E\left(w_{0} w_{k}^{\top}\right)$. Similar to Theorem 1 of Caner and Hansen (2001), we can show that

$$
\left(\frac{1}{\sqrt{n}} \sum_{t=1}^{\lfloor n s\rfloor} v_{t}, \frac{1}{\sqrt{n}} \sum_{t=1}^{\lfloor n s\rfloor} I_{t-1}^{*} u_{t}\right) \Longrightarrow\left[B_{v}(s), \sigma W(s, \lambda)\right] \text { in } D^{2}[0,1],
$$

where $W(s, \lambda)$ is a two-parameter standard Brownian motion on $(s, \lambda) \in[0,1]^{2}$ and $D[0,1]$ denotes the Skorohod space. The two-parameter Brownian motion is a special tool to derive the limiting distribution in threshold models.

We define the generalized F-type statistic as follows:

$$
\mathrm{GF}_{n}=\sup _{0 \leq k \leq k_{1}} \sup _{r_{1} \leq r \leq r_{2}} \mathrm{GF}_{n}(k, r), \quad \text { where } \mathrm{GF}_{n}(k, r)=n\left(\frac{\mathrm{RSS}_{0}-\mathrm{RSS}_{1}}{\mathrm{RSS}_{1}}\right),
$$


from which, we can see that this statistic is equivalent to $\operatorname{GLR}_{n}(k, r)$ in (2.5) in Section 2.1 if $x_{t}$ is stationary.

First, we consider the case that $x_{t}$ is unit-root, i.e., $\rho=1$, so that

$$
x_{t}=x_{t-1}+v_{t}
$$

The following theorem gives the asymptotic behavior of $\mathrm{GF}_{n}$ when $x_{t}$ is a unit-root process.

Theorem 2 If Assumptions 3-5 hold and $x_{t}$ is given by (2.8), then under $H_{0}$, it follows that

$$
\mathrm{GF}_{n} \stackrel{d}{\longrightarrow} \sup _{0 \leq \pi \leq \pi_{1}} \sup _{\lambda_{1} \leq \lambda \leq \lambda_{2}} \eta_{\pi \lambda}^{\top} N_{\pi \lambda}^{-1} \eta_{\pi \lambda}
$$

as $n \rightarrow \infty$, where $N_{\pi \lambda}=\lambda \Omega_{\pi}-\lambda^{2} \Omega_{\pi} \Omega_{0}^{-1} \Omega_{\pi}, \eta_{\pi \lambda}=\int_{\pi}^{1} \bar{B}_{v}(s) d W(s, \lambda)-\lambda \Omega_{\pi} \Omega_{0}^{-1} \int_{0}^{1} \bar{B}_{v}(s) d W(s, 1)$, and $\Omega_{\pi}=\int_{\pi}^{1} \bar{B}_{v}(s) \bar{B}_{v}^{\top}(s) d s, \bar{B}_{v}(s)=\left(1, B_{v}(s)\right)^{\top}$.

Next, we consider the case that $x_{t}$ is near-integrated, i.e.,

$$
x_{t}=\rho_{n} x_{t-1}+v_{t}, \rho_{n}=1-c / n, c>0 .
$$

We also make use of the diffusion process $K_{c}(s)=\int_{0}^{s} \mathrm{e}^{c(s-u)} d B_{v}(u)$ with $K_{c}(s)$ such that $d K_{c}(s)=$ $c K_{c}(s)+d B_{v}(s)$ and $K_{c}(0)=0$. Note that we can also write

$$
K_{c}(s)=B_{v}(s)+c \int_{0}^{s} \mathrm{e}^{c(s-u)} B_{v}(u) d u .
$$

It is obvious that when $c=0, K_{c}(s)$ becomes to $B_{v}(s)$ given in (2.6). More properties about $K_{c}(s)$ can be found in Section 3 of Phillips (1988). Under our assumptions, it follows directly from Lemma 3.1 in Phillips (1988) that $x_{\lfloor n s\rfloor} / \sqrt{n} \Longrightarrow K_{c}(s)$. The following theorem establishes the asymptotic behavior of $\mathrm{GF}_{n}$ when $x_{t}$ is a near-integrated process.

Theorem 3 If Assumptions 3-5 hold and $x_{t}$ is given by (2.9), then under $H_{0}$, we have

$$
\mathrm{GF}_{n} \stackrel{d}{\longrightarrow} \sup _{0 \leq \pi \leq \pi_{1}} \sup _{\lambda_{1} \leq \lambda \leq \lambda_{2}} \zeta_{\pi \lambda}^{\top} L_{\pi \lambda}^{-1} \zeta_{\pi \lambda}
$$

as $n \rightarrow \infty$, where $L_{\pi \lambda}=\lambda \Xi_{\pi}-\lambda^{2} \Xi_{\pi} \Xi_{0}^{-1} \Xi_{\pi}, \zeta_{\pi \lambda}=\int_{\pi}^{1} \bar{K}_{c}(s) d W(s, \lambda)-\lambda \Xi_{\pi} \Xi_{0}^{-1} \int_{0}^{1} \bar{K}_{c}(s) d W(s, 1)$, and $\Xi_{\pi}=\int_{\pi}^{1} \bar{K}_{c}(s) \bar{K}_{c}^{\top}(s) d s, \bar{K}_{c}(s)=\left(1, K_{c}(s)\right)^{\top}$.

Remark 1 When $c=0$, Theorem 3 includes Theorem 2 as a special case. When $\pi=0$, Theorem 3 also includes Proposition 1 in Gonzalo and Pitarakis (2012) as a special case because we have 
the same null hypothesis (no threshold and structural change) although the alternative hypothesis is different. Note that the formulation of our model (2.1) is different from model (1) in Gonzalo and Pitarakis (2012). Unlike the case with stationary predictor, $E\left(I_{t-1}^{*} X_{t} X_{t}^{\top}\right)$ cannot be simplified to $\lambda \Sigma$, when $x_{t}$ and $q_{t}$ are independent. A similar discussion about PR model without structural change was given by Gonzalo and Pitarakis (2012, p.232).

\section{Bootstrapped Critical Values}

We have seen in the previous section that structural change or non-stationarity affects the asymptotic distributions of the test statistics in complicated ways. Under the null, the test statistic has a limiting distribution given by a functional of a zero mean Gaussian process whose covariance function depends on the data generating process (DGP). The asymptotic critical values thus depend on the DGP and cannot be tabulated. In this section, we use a Bootstrap method to obtain the critical value for our tests, see Hansen (1996) and Zhu et al. (2014b) in different settings.

We first give some notations. Let $z_{t}(k, r)=\left(X_{t}^{\top}, I\left(q_{t} \leq r, t>k\right) X_{t}^{\top}\right)^{\top}$ and $u_{t}(\theta, k, r)=y_{t}-$ $z_{t}^{\top}(k, r) \theta$. Then,

$$
\theta_{n}(k, r) \equiv \arg \min _{\theta \in \Theta} \sum_{t=1}^{n} u_{t}^{2}(\theta, k, r)=\left(\widetilde{X}_{k r}^{\top} \widetilde{X}_{k r}\right)^{-1} \widetilde{X}_{k r}^{\top} Y,
$$

where $\widetilde{X}_{k r}=\left(X, X_{k r}\right)=\left(z_{1}(k, r), \ldots, z_{n}(k, r)\right)^{\top}$ and $\Theta$ is a compact parameter space of $\theta$. Assume that $\left\{\varepsilon_{t}\right\}_{t=1}^{n}$ is a sequence of i.i.d. $N(0,1)$ random variables. Let $\hat{u}=\left(\hat{u}_{1} \varepsilon_{1}, \ldots, \hat{u}_{n} \varepsilon_{n}\right)^{\top}$, where $\hat{u}_{t}=y_{t}-z_{t}^{\top}(k, r) \theta(k, r)$. By using $\left\{\hat{u}_{t}\right\}$, we will construct our Bootstrap statistics in this section.

\subsection{Stationary Predictor}

Denote $T_{k r}^{\star}=n^{-1 / 2}\left[X_{k r}^{\top}-X_{k r}^{\top} X\left(X^{\top} X\right)^{-1} X^{\top}\right] \hat{u}$. For each $(k, r)$, we define the Bootstrap GLR test statistic as follows:

$$
\widehat{\operatorname{GLR}}_{n}(k, r)=T_{k r}^{\star \top}\left\{\frac{X_{k r}^{\top} X_{k r}}{n}-\frac{X_{k r}^{\top} X}{n}\left(\frac{X^{\top} X}{n}\right)^{-1} \frac{X^{\top} X_{k r}}{n}\right\}^{-1} T_{k r}^{\star} / \hat{\sigma}_{n}^{2} .
$$

Note that $T_{k r}^{\star}$ can also be written as

$$
\left(-\frac{X_{k r}^{\top} X}{n}\left(\frac{X^{\top} X}{n}\right)^{-1}, I_{2}\right) \hat{Z}_{n}(k, r) \text { with } \hat{Z}_{n}(k, r)=\frac{1}{\sqrt{n}} \widetilde{X}_{k r}^{\top} \hat{u}=\frac{1}{\sqrt{n}} \sum_{t=1}^{n} z_{t}(k, r) \hat{u}_{t} \varepsilon_{t} .
$$


Our Bootstrap GLR test statistic is defined as follows:

$$
\widehat{\operatorname{GLR}}_{n} \equiv \sup _{0 \leq \pi \leq \pi_{1}} \sup _{\lambda_{1} \leq \lambda \leq \lambda_{2}} \widehat{\operatorname{GLR}}_{n}^{*}(\pi, \lambda) \equiv \sup _{0 \leq k \leq k_{1}} \sup _{r_{1} \leq r \leq r_{2}} \widehat{\operatorname{GLR}}_{n}(k, r)
$$

Before stating our result, we mention the concept 'weak convergence in probability', which generalizes convergence in distribution to allow for conditional (i.e. random) distribution functions. The asymptotic theory of $\widehat{\mathrm{GLR}}_{n}$ is stated in the following theorem, which says that the conditional limiting distribution is the same as the null distribution as in Theorem 1.

Theorem 4 If Assumptions 1-3 hold, then under $H_{0}$, it follows that

$$
\widehat{\operatorname{GLR}}_{n} \mid w_{1}^{*}, \ldots, w_{n}^{*} \stackrel{d}{\longrightarrow} \sup _{0 \leq \pi \leq \pi_{1}} \sup _{\lambda_{1} \leq \lambda \leq \lambda_{2}} \xi_{\pi \lambda}^{\top} M_{\pi \lambda}^{-1} \xi_{\pi \lambda}
$$

in probability as $n \rightarrow \infty$, where $w_{t}^{*}=\left(y_{t}, x_{t}, q_{t}\right), t=1, \ldots, n$.

From Theorem 3, conditional on the data sample $\left\{w_{1}^{*}, \ldots, w_{n}^{*}\right\}$, our Bootstrap procedure to obtain the critical value at significance level $\alpha$ is as follows:

(i) generate i.i.d. $N(0,1)$ samples $\left\{\varepsilon_{t}\right\}_{t=1}^{n}$ and calculate $\widehat{\mathrm{GLR}}_{n}$ via $(3.1)$;

(ii) repeat step (i) $J$ times to get $\left\{\widehat{\operatorname{GLR}}_{n}^{(1)}, \ldots, \widehat{\operatorname{GLR}}_{n}^{(J)}\right\}$;

(iii) choose $c_{n, \alpha}^{J}$ as the $\alpha$-th upper percentile of $\left\{\widehat{\operatorname{GLR}}_{n}^{(1)}, \ldots, \widehat{\operatorname{GLR}}_{n}^{(J)}\right\}$.

The critical value for the GLR test is $c_{n, \alpha}^{J}$. The following corollary guarantees that our Bootstrapped critical value $c_{n, \alpha}^{J}$ is asymptotically valid, which is called unconditional Bootstrap validity and its proof is similar to that for Corollary 2 in Zhu et al. (2014b) and hence is omitted.

Corollary 2 If Assumptions 1-3 hold, under $H_{0}$, we have $\lim _{n \rightarrow \infty} \lim _{J \rightarrow \infty} P\left(\mathrm{GLR}_{n} \geq c_{n, \alpha}^{J}\right)=\alpha$.

\subsection{Unit Root Predictor}

It is well known that standard Bootstrap techniques fail in the context of non-stationary AR models. One has to employ the subsample Bootstrap method which faces the difficult issue of choosing the subsample size. To allow not-stationarity or an unknown degree of persistence in the predictors, Hansen (2000) proposed the fixed regressor Bootstrap method to obtain critical value. Georgiev et al. $(2018,2019)$ established the validity of this Bootstrap method for test statistics for the parameter instability in predictive regression models.

Using a similar idea as that in Section 3.1, we construct the Bootstrap GF test as follows:

$$
\widehat{\mathrm{GF}}_{n}=\sup _{0 \leq k \leq k_{1}} \sup _{r_{1} \leq r \leq r_{2}} T_{k r}^{\star \top} R_{k r}^{-1} T_{k r}^{\star} / \hat{\sigma}_{n}^{2}(k, r)
$$


where $T_{k r}^{\star}=D_{n} X_{k r}^{\top} \hat{u}-D_{n} X_{k r}^{\top} X D_{n}\left(D_{n} X^{\top} X D_{n}\right)^{-1} D_{n} X^{\top} \hat{u}$ and $R_{k r}$ is given in the proof of Theorem 2, i.e., $R_{k r}=D_{n} X_{k r}^{\top} X_{k r} D_{n}-D_{n} X_{k r}^{\top} X D_{n}\left(D_{n} X^{\top} X D_{n}\right)^{-1} D_{n} X^{\top} X_{k r} D_{n}$ and $D_{n}=\operatorname{diag}\{1 / \sqrt{n}, 1 / n\}$. To formulate a useful asymptotic result, a weaker convergence mode is required, i.e., the terminology of weak convergence of random measures, which is weaker than weak convergence in probability. It reduces to the latter when the limit distribution is non-random. For the definition of this terminology and its application to Bootstrap, we refer to the paper by Cavaliere and Georgiev (2020). Following Georgiev et al. (2019), we need to strengthen Assumption 4 as follows:

Assumption 6 Assumption 4 holds together with the following conditions: (a) wt is drawn from a doubly infinite strictly stationary and ergodic sequence $\left\{w_{t}\right\}_{t=-\infty}^{\infty}$ which is a MDS with respect to its own past. (b) $\left\{u_{t}\right\}_{t=-\infty}^{\infty}$ is a MDS also with respect to $\mathcal{G}_{t}^{1} \vee \mathcal{G}_{t}^{2}$, where $\mathcal{G}_{t}^{1}$ and $\mathcal{G}_{t}^{2}$ are the $\sigma$-algebras generated by $\left\{v_{t}\right\}_{t=-\infty}^{\infty}$ and $\left\{u_{t}\right\}_{t=-\infty}^{\infty}$, respectively, and $\mathcal{G}_{t}^{1} \vee \mathcal{G}_{t}^{2}$ denotes the smallest $\sigma$-algebra containing both $\mathcal{G}_{t}^{1}$ and $\mathcal{G}_{t}^{2}$.

The asymptotic theory of our Bootstrapped statistic $\widehat{\mathrm{GF}}_{n}$ is given as follows, which is different from Theorem 4.

Theorem 5 (a). If Assumptions 3-6 hold and $x_{t}$ is given by (2.8), then under $H_{0}$, it follows that

$$
\widehat{\mathrm{GF}}_{n}\left|w_{1}^{*}, \ldots, w_{n}^{*} \longrightarrow \sup _{0 \leq \pi \leq \pi_{1}} \sup _{\lambda_{1} \leq \lambda \leq \lambda_{2}} \eta_{\pi \lambda}^{\top} N_{\pi \lambda}^{-1} \eta_{\pi \lambda}\right| B_{v}
$$

as $n \rightarrow \infty$, where $w_{t}^{*}=\left(y_{t}, x_{t}, q_{t}\right), t=1, \ldots, n$ and $B_{v}$ is given in (2.6). (b) if Assumptions 3-6 hold and $x_{t}$ is given by (2.9), then under $H_{0}$, it follows that

$$
\widehat{\mathrm{GF}}_{n}\left|w_{1}^{*}, \ldots, w_{n}^{*} \longrightarrow \sup _{0 \leq \pi \leq \pi_{1}} \sup _{\lambda_{1} \leq \lambda \leq \lambda_{2}} \zeta_{\pi \lambda}^{\top} L_{\pi \lambda}^{-1} \zeta_{\pi \lambda}\right| B_{v}
$$

as $n \rightarrow \infty$.

Similar to the Bootstrap procedure in Section 3.1, we first obtain the Bootstrap sample $\left\{\hat{W}_{n}^{(1)}, \ldots\right.$, $\left.\hat{W}_{n}^{(J)}\right\}$ and then use its $\alpha$-th upper percentile $d_{n, \alpha}^{J}$ as the approximating critical value of the GF test statistic $\mathrm{GF}_{n}$. The following corollary guarantees that our Bootstrapped critical value $d_{n, \alpha}^{J}$ is asymptotically valid, which is called unconditional Bootstrap validity and its proof is similar to that for Corollary 1 in Georgiev et al. (2019) and hence is omitted.

Corollary 3 If Assumptions 3-6 hold, under $H_{0}$, then $\lim _{n \rightarrow \infty} \lim _{J \rightarrow \infty} P\left(\mathrm{GF}_{n} \geq d_{n, \alpha}^{J} \mid w_{1}^{*}, \ldots, w_{n}^{*}\right)=\alpha$. 


\section{Simulation Studies}

In this section, we report the performances of $\mathrm{GLR}_{n}$ and $\mathrm{GF}_{n}$ in stationary and unit root cases in the finite sample, respectively.

We first study the size of two tests at the nominal $1 \%$ and $5 \%$ level, respectively. The data is generated from the null model $(2.1)$ with $\left(\psi_{0}, \psi_{1}\right)=(0.1,0.2)$ and $k=n$, where the predictor $x_{t}$ is modeled as $x_{t}=\rho x_{t-1}+\nu_{t}$ with $\rho=0.2,0.9$, and 1 and $\nu_{t} \sim N(0,1)$, and the threshold variable $q_{t} \sim N(0,1)$. Based on the Bootstrapped critical values, we consider rejection frequencies from 5000 replications with the sample sizes 200, 500 and 1000. The results are presented in Table 1. We find that the sizes of the tests are acceptable no matter in stationary or unit root cases. Additionally, both $\mathrm{GLR}_{n}$ and $\mathrm{GF}_{n}$ perform better when the sample size is not less than 500 .

Table 1: Sizes of $\mathrm{GLR}_{n}$ and $\mathrm{GF}_{n}$ under the null model (2.1).

\begin{tabular}{|c|c|c|c|c|c|c|c|}
\hline & \multirow[b]{2}{*}{$n$} & \multicolumn{3}{|c|}{$\alpha=0.01$} & \multicolumn{3}{|c|}{$\alpha=0.05$} \\
\hline & & 200 & 500 & 1000 & 200 & 500 & 1000 \\
\hline \multirow{2}{*}{ stationary case $\left(\mathrm{GLR}_{n}\right)$} & $\rho=0.2$ & 1.17 & 0.90 & 1.05 & 5.23 & 5.12 & 5.03 \\
\hline & $\rho=0.9$ & 0.82 & 1.08 & 1.04 & 4.81 & 4.92 & 5.01 \\
\hline unit root case $\left(\mathrm{GF}_{n}\right)$ & $\rho=1$ & 1.22 & 1.07 & 0.99 & 5.20 & 4.96 & 5.01 \\
\hline
\end{tabular}

Next, in the stationary cases with $\rho=0.2$ and 0.9 , we explore the power of the GLR $_{n}$ test against different alternative models, where $\phi_{0}$ and $\phi_{1}$ take values in $\{-2,-1,-0.6,0.6,1,2\}$. In the non-stationary cases with $\rho=1$, the power of $\mathrm{GF}_{n}$ test against different alternative models is considered, where $\phi_{0}$ and $\phi_{1}$ take values in $\{-1,-0.6,-0.2,0.2,0.6,1\}$. At the same time, we consider whether our tests will be affected by the time of change $k^{*}$ and the true threshold $r$ in the stationary and non-stationary cases, i.e., $\left(k^{*}, r\right)=(100,0),(150,0)$, and $(150,0.2)$. The results of the stationary and unit root cases are summarized in Table 2 and Table 3, respectively. The first point we should pay attention is that the farther $\left(\phi_{0}, \phi_{1}\right)$ away from $(0,0)$, the stronger the power. But the power is seriously affected by $\phi_{1}$. This is reasonable since the predictor $x_{t}$ plays an important in the BR model. The second is that different $k^{*}$ and $r$ do not cause huge differences in power, which implies that our test is applicable and credible when the time of change and threshold 
Table 2: Power against model $(2.1)$ with $\left(\psi_{0}, \psi_{1}\right)=(0.1,0,2)$ and $x_{t}=0.9 x_{t-1}+\nu_{t}$.

\begin{tabular}{|c|c|c|c|c|c|c|c|c|c|c|c|c|c|}
\hline \multicolumn{7}{|c|}{$\alpha=0.01$} & \multicolumn{7}{|c|}{$\alpha=0.05$} \\
\hline & \multicolumn{6}{|c|}{$k^{*}=100, r=0$} & \multicolumn{7}{|c|}{$k^{*}=100, r=0$} \\
\hline $\begin{array}{r}\phi_{0} \\
\phi_{1}\end{array}$ & $\underline{-2.0}$ & $\underline{-1.0}$ & $\underline{-0.6}$ & $\underline{0.6}$ & $\underline{1.0}$ & $\underline{2.0}$ & $\begin{array}{c}\phi_{0} \\
\phi_{1}\end{array}$ & $\underline{-2.0}$ & $\underline{-1.0}$ & $\underline{-0.6}$ & $\underline{0.6}$ & $\underline{1.0}$ & $\underline{2.0}$ \\
\hline$\underline{-2.0}$ & 57.4 & 56.3 & 54.5 & 54.6 & 56.7 & 56.9 & $\underline{-2.0}$ & 80.5 & 79.0 & 78.1 & 77.6 & 79.4 & 79.9 \\
\hline$\underline{-1.0}$ & 23.9 & 20.4 & 17.8 & 16.9 & 19.7 & 24.0 & $\underline{-1.0}$ & 46.0 & 38.6 & 36.9 & 37.9 & 38.8 & 45.7 \\
\hline$\underline{-0.6}$ & 8.4 & 5.0 & 4.7 & 4.3 & 4.8 & 8.7 & $\underline{-0.6}$ & 17.1 & 14.3 & 13.7 & 13.5 & 14.1 & 16.9 \\
\hline$\underline{0.6}$ & 8.6 & 4.9 & 4.5 & 4.2 & 4.6 & 8.3 & $\underline{0.6}$ & 17.0 & 14.1 & 13.8 & 13.7 & 14.2 & 17.2 \\
\hline$\underline{1.0}$ & 23.6 & 20.1 & 16.9 & 17.6 & 20.2 & 24.0 & $\underline{1.0}$ & 46.2 & 38.7 & 37.3 & 37.2 & 38.9 & 46.5 \\
\hline \multirow[t]{2}{*}{$\underline{2.0}$} & 57.2 & 56.2 & 54.0 & 54.3 & 56.7 & 57.0 & $\underline{2.0}$ & 80.2 & 79.1 & 78.0 & 77.8 & 79.3 & 80.0 \\
\hline & \multicolumn{6}{|c|}{$k^{*}=150, r=0$} & & \multicolumn{6}{|c|}{$k^{*}=150, r=0$} \\
\hline$\phi_{1}$ & -2.0 & $\underline{-1.0}$ & $\underline{-0.6}$ & $\underline{0.6}$ & $\underline{1.0}$ & $\underline{2.0}$ & $\begin{array}{r}\phi_{0} \\
\phi_{1}\end{array}$ & -2.0 & -1.0 & $\underline{-0.6}$ & $\underline{0.6}$ & $\underline{1.0}$ & $\underline{2.0}$ \\
\hline$\underline{-2.0}$ & 57.2 & 56.2 & 54.1 & 54.5 & 56.8 & 57.3 & $\underline{-2.0}$ & 80.5 & 78.7 & 78.4 & 77.9 & 79.2 & 80.4 \\
\hline$\underline{-1.0}$ & 24.2 & 19.7 & 17.6 & 17.4 & 20.6 & 24.4 & -1.0 & 45.9 & 38.8 & 37.2 & 38.0 & 39.2 & 46.3 \\
\hline$\underline{-0.6}$ & 8.5 & 4.8 & 4.7 & 4.4 & 4.7 & 8.5 & $\underline{-0.6}$ & 17.2 & 14.2 & 13.6 & 13.8 & 13.9 & 17.3 \\
\hline$\underline{0.6}$ & 8.3 & 4.8 & 4.5 & 4.3 & 4.5 & 8.7 & $\underline{0.6}$ & 17.1 & 14.0 & 13.5 & 13.6 & 14.3 & 17.2 \\
\hline$\underline{1.0}$ & 24.0 & 20.3 & 16.7 & 17.9 & 20.4 & 23.8 & $\underline{1.0}$ & 46.2 & 38.9 & 37.4 & 37.4 & 39.3 & 46.0 \\
\hline \multirow[t]{2}{*}{$\underline{2.0}$} & 57.0 & 56.4 & 54.3 & 54.4 & 56.7 & 57.1 & $\underline{2.0}$ & 80.3 & 79.0 & 78.3 & 77.4 & 79.1 & 79.8 \\
\hline & \multicolumn{6}{|c|}{$k^{*}=150, r=0.2$} & & \multicolumn{6}{|c|}{$k^{*}=150, r=0.2$} \\
\hline$\phi_{1}$ & $\underline{-2.0}$ & -1.0 & $\underline{-0.6}$ & $\underline{0.6}$ & $\underline{1.0}$ & $\underline{2.0}$ & $\begin{array}{c}\phi_{0} \\
\phi_{1}\end{array}$ & -2.0 & -1.0 & $\underline{-0.6}$ & $\underline{0.6}$ & $\underline{1.0}$ & $\underline{2.0}$ \\
\hline$\underline{-2.0}$ & 56.5 & 56.0 & 53.9 & 53.7 & 55.8 & 56.3 & $\underline{-2.0}$ & 79.2 & 77.8 & 77.2 & 76.9 & 78.0 & 78.9 \\
\hline$\underline{-1.0}$ & 23.2 & 19.0 & 16.7 & 15.7 & 18.6 & 23.5 & -1.0 & 44.8 & 37.4 & 34.8 & 35.7 & 37.8 & 44.9 \\
\hline$\underline{-0.6}$ & 7.9 & 4.8 & 4.4 & 4.3 & 4.6 & 7.8 & $\underline{-0.6}$ & 16.0 & 13.3 & 12.4 & 12.5 & 13.8 & 16.2 \\
\hline$\underline{0.6}$ & 8.0 & 4.5 & 4.1 & 4.0 & 4.4 & 7.9 & $\underline{0.6}$ & 16.2 & 13.4 & 12.7 & 12.8 & 13.7 & 16.1 \\
\hline$\underline{1.0}$ & 23.0 & 19.1 & 15.8 & 15.6 & 19.4 & 23.1 & $\underline{1.0}$ & 45.3 & 37.2 & 35.4 & 35.6 & 37.4 & 45.2 \\
\hline$\underline{2.0}$ & 56.3 & 56.1 & 54.0 & 53.7 & 56.0 & 56.4 & $\underline{2.0}$ & 79.3 & 77.6 & 77.0 & 76.8 & 78.2 & 79.0 \\
\hline
\end{tabular}


Table 3: Power against model $(2.1)$ with $\left(\psi_{0}, \psi_{1}\right)=(0.1,0,2)$ and $x_{t}=x_{t-1}+\nu_{t}$.

\begin{tabular}{|c|c|c|c|c|c|c|c|c|c|c|c|c|c|}
\hline \multicolumn{7}{|c|}{$\alpha=0.01$} & \multicolumn{7}{|c|}{$\alpha=0.05$} \\
\hline & \multicolumn{6}{|c|}{$k^{*}=100, r=0$} & \multicolumn{7}{|c|}{$k^{*}=100, r=0$} \\
\hline $\begin{array}{c}\phi_{0} \\
\phi_{1}\end{array}$ & $\underline{-1.0}$ & $\underline{-0.6}$ & -0.2 & $\underline{0.2}$ & $\underline{0.6}$ & $\underline{1.0}$ & $\begin{array}{r}\phi_{0} \\
\phi_{1}\end{array}$ & $\underline{-1.0}$ & $\underline{-0.6}$ & $\underline{-0.2}$ & $\underline{0.2}$ & $\underline{0.6}$ & $\underline{1.0}$ \\
\hline$\underline{-1.0}$ & 85.8 & 84.9 & 84.7 & 84.1 & 84.6 & 86.2 & $\underline{-1.0}$ & 99.1 & 97.8 & 97.3 & 97.2 & 98.0 & 99.4 \\
\hline$\underline{-0.6}$ & 53.7 & 51.9 & 50.4 & 50.1 & 51.6 & 54.0 & $\underline{-0.6}$ & 73.2 & 72.3 & 72.6 & 71.7 & 72.5 & 73.1 \\
\hline$\underline{-0.2}$ & 4.9 & 3.9 & 2.5 & 2.7 & 3.8 & 4.7 & $\underline{-0.2}$ & 10.4 & 9.6 & 8.5 & 8.9 & 9.5 & 10.7 \\
\hline$\underline{0.2}$ & 5.0 & 4.4 & 2.4 & 2.8 & 3.6 & 4.9 & $\underline{0.2}$ & 10.8 & 9.7 & 8.4 & 8.7 & 9.6 & 10.5 \\
\hline$\underline{0.6}$ & 53.8 & 52.6 & 51.4 & 50.6 & 52.8 & 53.4 & $\underline{0.6}$ & 73.6 & 72.4 & 71.9 & 71.6 & 72.4 & 73.0 \\
\hline$\underline{1.0}$ & 85.6 & 84.2 & 84.4 & 84.6 & 84.2 & 86.8 & $\underline{1.0}$ & 99.3 & 98.5 & 97.2 & 97.4 & 98.9 & 99.6 \\
\hline & \multicolumn{6}{|c|}{$k^{*}=150, r=0$} & & \multicolumn{6}{|c|}{$k^{*}=150, r=0$} \\
\hline $\begin{array}{c}\phi_{0} \\
\phi_{1}\end{array}$ & $\underline{-1.0}$ & $\underline{-0.6}$ & $\underline{-0.2}$ & $\underline{0.2}$ & $\underline{0.6}$ & $\underline{1.0}$ & $\begin{array}{r}\phi_{0} \\
\phi_{1}\end{array}$ & $\underline{-1.0}$ & $\underline{-0.6}$ & $\underline{-0.2}$ & $\underline{0.2}$ & $\underline{0.6}$ & $\underline{1.0}$ \\
\hline$\underline{-1.0}$ & 85.7 & 85.0 & 84.5 & 84.3 & 84.8 & 86.9 & $\underline{-1.0}$ & 99.2 & 98.7 & 97.4 & 97.3 & 98.8 & 99.6 \\
\hline$\underline{-0.6}$ & 53.0 & 52.6 & 51.9 & 51.2 & 52.0 & 54.6 & $\underline{-0.6}$ & 73.4 & 72.5 & 71.8 & 71.4 & 72.6 & 73.0 \\
\hline$\underline{-0.2}$ & 5.0 & 3.8 & 2.8 & 2.6 & 4.2 & 4.9 & $\underline{-0.2}$ & 10.7 & 9.4 & 8.7 & 8.8 & 9.7 & 10.6 \\
\hline$\underline{0.2}$ & 5.1 & 4.0 & 2.9 & 2.8 & 3.9 & 4.9 & $\underline{0.2}$ & 10.5 & 9.7 & 8.6 & 8.7 & 9.6 & 10.8 \\
\hline$\underline{0.6}$ & 53.9 & 52.5 & 50.2 & 50.3 & 52.4 & 54.2 & $\underline{0.6}$ & 73.6 & 72.1 & 72.4 & 71.6 & 72.8 & 73.9 \\
\hline \multirow[t]{2}{*}{$\underline{1.0}$} & 85.7 & 85.6 & 84.3 & 84.5 & 84.8 & 86.4 & $\underline{1.0}$ & 99.1 & 98.9 & 97.3 & 97.2 & 98.8 & 99.7 \\
\hline & \multicolumn{6}{|c|}{$k^{*}=150, r=0.2$} & & \multicolumn{6}{|c|}{$k^{*}=150, r=0.2$} \\
\hline $\begin{array}{c}\phi_{0} \\
\phi_{1}\end{array}$ & $\underline{-1.0}$ & $\underline{-0.6}$ & $\underline{-0.2}$ & $\underline{0.2}$ & $\underline{0.6}$ & $\underline{1.0}$ & $\begin{array}{r}\phi_{0} \\
\phi_{1}\end{array}$ & $\underline{-1.0}$ & $\underline{-0.6}$ & $\underline{-0.2}$ & $\underline{0.2}$ & $\underline{0.6}$ & $\underline{1.0}$ \\
\hline$\underline{-1.0}$ & 83.7 & 82.6 & 81.1 & 80.9 & 82.4 & 83.6 & $\underline{-1.0}$ & 97.1 & 96.7 & 96.4 & 96.0 & 96.5 & 97.1 \\
\hline$\underline{-0.6}$ & 51.3 & 50.5 & 49.4 & 48.7 & 50.6 & 51.4 & $\underline{-0.6}$ & 72.0 & 70.2 & 69.7 & 69.4 & 69.8 & 72.1 \\
\hline$\underline{-0.2}$ & 4.1 & 3.0 & 2.0 & 1.9 & 2.9 & 3.8 & $\underline{-0.2}$ & 9.3 & 8.7 & 7.4 & 7.8 & 8.5 & 9.2 \\
\hline$\underline{0.2}$ & 4.1 & 3.5 & 2.1 & 2.2 & 3.0 & 3.8 & $\underline{0.2}$ & 9.5 & 8.6 & 7.2 & 7.4 & 8.8 & 9.3 \\
\hline$\underline{0.6}$ & 51.8 & 50.3 & 48.2 & 48.4 & 50.2 & 51.6 & $\underline{0.6}$ & 72.0 & 69.8 & 69.8 & 69.6 & 70.2 & 71.9 \\
\hline$\underline{1.0}$ & 83.9 & 82.6 & 80.9 & 80.8 & 82.3 & 83.8 & $\underline{1.0}$ & 97.1 & 96.5 & 95.6 & 96.2 & 96.4 & 97.0 \\
\hline
\end{tabular}


are unknown.

\section{A Real Example}

In this section, $\mathrm{GLR}_{n}$ and $\mathrm{GF}_{n}$ are applied to test whether commonly used financial variables have threshold effects and change points with respect to excess stock market returns. We consider the data sets analyzed in Welch and Goyal (2008) and Kostakis et al. (2015), but the period of data is 1927-2019, which is updated by Amit Goyal's Web. Following the setting in Welch and Goyal (2008), S\&P 500 value-weighted log excess returns are used as excess market returns. We consider the following six variables as predictors: book-to-market value ratio $(\mathbf{b} / \mathbf{m})$, T-bill rate (tbl), net equity expansion (ntis), inflation rate (inf), long-term return (ltr), stock variance (svar), which are monthly or quarterly.

To determine if we should use $\mathrm{GLR}_{n}$ or $\mathrm{GF}_{n}$, we first perform unit root tests for these six predictors and the corresponding $p$-values are presented in Table 4. From Table 4, one can find that only the monthly or quarterly T-bill rate is a unit root process at the significance level 0.05. Thus, we use $\mathrm{GF}_{n}$ to test the null in (2.2) when $x_{t}$ is the T-bill rate, and use GLR $\mathrm{GL}_{n}$ test for the null in (2.2) when $x_{t}$ is other five predictors. The results are reported in Table 4 , from which, one can observe that $\mathrm{GF}_{n}$ rejects the null in (2.2) at the level 0.01 and 0.05 when $x_{t}$ is the predictor as monthly data and at the level 0.01 when $x_{t}$ is the predictor as quarterly data. When the predication variable $x_{t}$ is book-to-market value ratio or stock variance as both monthly and quarterly data, $\mathrm{GLR}_{n}$ test rejects the null in (2.2) at both level 0.01 and 0.05 . When $x_{t}$ is inflation rate or long-term return as both monthly and quarterly data, $\mathrm{GLR}_{n}$ cannot reject the null in (2.2) at both level 0.01 and 0.05. When $x_{t}$ is net equity expansion, except for the monthly data at the level $0.01, G L R_{n}$ test cannot reject the null in (2.2). This example illustrates that our test can be used for testing the change-point and threshold effect in PR model simultaneously.

\section{Conclusion}

In this paper, two tests, termed as generalized likelihood ratio (GLR) and generalized F (GF), are proposed to testing structural changes and thresholds in linear predictive regression models. The

former is for the case that regressor is stationary and the latter is for the situation that regressor 
Table 4: $\mathrm{GLR}_{n}$ and $\mathrm{GF}_{n}$ Tests for six predictive regressors.

\begin{tabular}{|c|c|c|c|c|c|c|}
\hline & \multicolumn{3}{|c|}{ monthly } & \multicolumn{3}{|c|}{ quarterly } \\
\hline & \multirow{2}{*}{$\begin{array}{c}\mathrm{p} \text {-value for } \\
\text { unit-root test }\end{array}$} & \multicolumn{2}{|c|}{$\alpha$} & \multirow{2}{*}{$\begin{array}{c}\mathrm{p} \text {-value for } \\
\text { unit-root test }\end{array}$} & \multicolumn{2}{|c|}{$\alpha$} \\
\hline & & 0.01 & 0.05 & & 0.01 & 0.05 \\
\hline $\mathbf{b} / \mathbf{m}$ (book to market) & $<0.01$ & $1^{* *}$ & 1 & 0.0281 & 1 & 1 \\
\hline tbl (T-bill rate) & 0.4574 & 1 & 1 & 0.5293 & 1 & 0 \\
\hline ntis (net equity expansion) & $<0.01$ & 1 & 0 & $<0.01$ & 0 & 0 \\
\hline infl (inflation) & $<0.01$ & 0 & 0 & $<0.01$ & 0 & 0 \\
\hline ltr (long term return) & $<0.01$ & 0 & 0 & $<0.01$ & 0 & 0 \\
\hline svar (stock variance) & $<0.01$ & 1 & 1 & $<0.01$ & 1 & 1 \\
\hline
\end{tabular}

is nonstationary such as unit root process or nearly integrated process. The limiting distribution of the proposed test statistics are derived under both the null and alternative hypotheses. It turns out that the limiting distributions are functionals of some Gaussian processes with complicated covariance structures and depend heavily on the characteristics of regress, in the sense that regressor is stationary or nonstationary. Due to the complexity of the limiting distributions of the proposed test statistics, a Bootstrap approach is suggested for computing the critical values of the proposed tests.

Finally, we note several possible extensions of the present study. For example, it may be of interest to have some unified testing procedures free of the characteristics of regressor (regardless of the persistence of regressor). Also, it might allow regressor to have structural changes or thresholds too. Further, in Assumption 4 , if $\operatorname{Cov}\left(u_{t}, v_{t}\right) \neq 0$, which is the so called "embedded endogeneity", then the results for the unit root predictor may be invalid, then we can relax this assumption by adopting the so called "leads and lags" approach in Section 4 in Chen (2015) or consider the weighted method in Cai et al. (2020). We leave such extensions as possible future research topics. 


\section{Acknowledgements}

Zhu's work is supported by National Natural Science Foundation of China (NSFC) Grants (11871027 and 11731015) and Cultivation Plan for Excellent Young Scholar Candidates of Jilin University. Ling's research was supported by Hong Kong Research Grants Commission Grants (16303118 and 16301620), RGC-Theme-based Research Scheme, NSFC (No.11731015) and Australian Research Council. Cai's research is partially supported by NSFC Grants (71631004, 72033008 and 71131008).

\section{References}

[1] Andrews, D.W.K. (1993). Tests for parameter instability and structural change with unknown change point. Econometrica, 61, 821-856.

[2] Bai, J. and Perron, P. (1998). Estimating and testing linear models with multiple structural changes. Econometrica, 66, 47-78.

[3] Berkes, I., Horváth, L., Ling, S. and Schauer, J. (2011). Testing for structural change of AR model to threshold AR model.Journal of Time Series Analysis, 32, 547-565.

[4] Breitung, J. and Demetrescu, M. (2015). Instrumental variable and variable addition based inference in predictive regressions. Journal of Econometrics, 187, 358-375.

[5] Cai, Z., Chen, H. and Liao, X. (2020). A new robust inference for predictive quantile regression. Working paper.

[6] Cai, Z., Fang, Y., Lin, M. and Su, J. (2019). Inferences for a partially varying coefficient model with endogenous regressors. Journal of Business $\& 3$ Economic Statistics, 37, 158-170.

[7] Cai, Z. and Tiwari, R.C. (2000). Application of a local linear autoregressive model to BOD time series. Environmetrics, 11, 341-350.

[8] Cai, Z. and Wang, Y. (2014). Testing predictive regression models with nonstationary regressors. Journal of Econometrics, 178, 4-14.

[9] Cai, Z., Wang, Y. and Wang, Y. (2015). Testing instability in a predictive regression model with nonstationary regressors. Econometric Theory, 31, 953-980.

[10] Campbell, J.Y. and Yogo, M. (2006). Efficient tests of stock return predictability. Journal of Financial Economics, 81, 27-60. 
[11] Caner, M. and Hansen, B.E. (2001). Threshold autoregression with a unit root. Econometrica, 69, $1555-1596$.

[12] Cavaliere, G. and Georgiev, I. (2020). Inference under random limit bootstrap measures. Econometrica, 88, 2547-2574.

[13] Chan, K.S. (1990). Testing for threshold autoregression. Annals of Statistics, 18, 1886-1894.

[14] Chen, H. (2015). Robust estimation and inference for threshold models with integrated regressors. Econometric Theory, 31, 778-810.

[15] Chow, G.C. (1960). Tests of equality between sets of coefficients in two linear regressions. Econometrica, 28, 591-605.

[16] Georgiev, I., Harvey, D.I., Leybourne, S.J. and Taylor, A.M.R. (2018). Testing for parameter instability in predictive regression models. Journal of Econometrics, 204, 101-118.

[17] Georgiev, I., Harvey, D.I., Leybourne, S.J. and Taylor, A.M.R. (2019). A Bootstrap stationarity test for predictive regression invalidity. Journal of Business 83 Economic Statistics, 37, 528-541.

[18] Gonzalo, J. and Pitarakis, J. (2012). Regime-specific predictability in predictive regressions. Journal of Business 63 Economic Statistics, 30, 229-241.

[19] Gonzalo, J. and Pitarakis, J. (2017). Inferring the predictability induced by a persistent regressor in a predictive threshold model. Journal of Business 83 Economic Statistics, 35, 202-217.

[20] Hansen, B.E. (1996). Inference when a nuisance parameter is not indentified under the null hypothesis. Econometrica, 64, 413-430.

[21] Hansen, B.E. (2000). Testing for structural change in conditional models. Journal of Econometrics, 97, 93-115.

[22] Horváth, L. (1993). The maximum likelihood method for testing changes in the parameters of normal observations. Annals of Statistics — bf 21 671-680.

[23] Jansson, M. and Moreira, M.J. (2006). Optimal inference in regression models with nearly integrated regressors. Econometrica, 74, 681-714.

[24] Kostakis, A., Magdalinos, T. and Stamatogiannis, M.P. (2015). Robust econometric inference for stock return predictability. Review of Financial Studies, 28, 1506-1553.

[25] Li, D., Ling, S. and Zhang, R. (2016). On a threshold double autoregressive model. Journal of Business E Economic Statistics, 34, 68-80. 
[26] Liao, X., Cai, Z. and Chen, H. (2018). A perspective on recent methods on testing predictability of asset returns. Applied Mathematics-A Journal of Chinese Universities, 33, 127-144.

[27] Ling, S. (2007). Testing for change points in time series models and limiting theorems for NED sequences. Annals of Statistics, 35, 1213-1237.

[28] Phillips, P.C.B. (1988). Regression theory for near-integrated time series. Econometrica, 56, 1021-1043.

[29] Pitarakis, J. (2017). A simple approach for diagnosing instabilities in predictive regressions. Oxford Bulletin of Economics and Statistics, 79, 851-874.

[30] Quandt, R.E. (1960). Tests of the hypothesis that a linear regression system obeys two separate regimes. Journal of the American Statistical Association, 55, 324-330.

[31] Schott, J.R. (2017). Matrix Analysis for Statistics(3rd ed). New Jersey: Wiley.

[32] Welch, I. and Goyal, A. (2008). A comprehensive look at the empirical performance of equity premium prediction. Review of Financial Studies, 21, 1455-1508.

[33] Wong, C.S. and Li, W.K. (1997). Testing for threshold autoregression with conditional heteroscedasticity. Biometrika, 84, 407-418.

[34] Yang, B., Liu, X., Peng, L. and Cai, Z. (2021). Unified tests for a dynamic predictive regression. Journal of Business \&3 Economic Statistics with DOI: https://doi.org/10.1080/07350015.2020.1714632.

[35] Yang, B., Long, W., Peng, L. and Cai, Z. (2020). Testing the predictability of U.S. housing price index returns based on an IVX-AR model. Journal of the American Statistical Association, 115, 1598-1619.

[36] Zhu, F., Cai, Z. and Peng, L. (2014a). Predictive regressions for macroeconomic data. Annals of Applied Statistics, 8, 577-594.

[37] Zhu, K. and Ling, S. (2012). Likelihood ratio tests for the structural change of an $\operatorname{AR}(p)$ model to a threshold AR $(p)$ model. Journal of Time Series Analysis, 33, 223-232.

[38] Zhu, K., Yu, P.L.H. and Li, W.K. (2014b). Testing for the buffered autoregressive processes. Statistica Sinica, 24, 971-984. 


\section{Appendix: Mathematical Proofs}

Proof of Theorem 1: Using a two-term Taylor expansion, we have

$$
\operatorname{GLR}_{n}(k, r)=S_{n}(k, r) / \hat{\sigma}_{n}^{2}+S_{n}^{2}(k, r) / 2 n \xi_{n}^{2}(k, r),
$$

where $S_{n}(k, r)=n\left(\hat{\sigma}_{n}^{2}-\hat{\sigma}_{n}^{2}(k, r)\right)$ and $\xi_{n}^{2}(k, r)$ is between $\hat{\sigma}_{n}^{2}$ and $\hat{\sigma}_{n}^{2}(k, r)$. By (2.3) and (2.4), we can show that (for example, see Example 7.1 in Schott (2017))

$$
S_{n}(k, r)=T_{k r}^{\top}\left\{\frac{X_{k r}^{\top} X_{k r}}{n}-\frac{X_{k r}^{\top} X}{n}\left(\frac{X^{\top} X}{n}\right)^{-1} \frac{X^{\top} X_{k r}}{n}\right\}^{-1} T_{k r},
$$

where $T_{k r}=n^{-1 / 2}\left[X_{k r}^{\top}-X_{k r}^{\top} X\left(X^{\top} X\right)^{-1} X^{\top}\right] Y$. Note that $T_{k r}=n^{-1 / 2}\left[X_{k r}^{\top}-X_{k r}^{\top} X\left(X^{\top} X\right)^{-1} X^{\top}\right] u$ if $H_{0}$ holds. Re-scale the time axis by setting $k=\lfloor n \pi\rfloor$ with $\pi \in[0,1]$, and denote $X_{\pi \lambda}^{*}=X_{\lfloor n \pi\rfloor, r}$ and $T_{\pi \lambda}^{*}=T_{\lfloor n \pi\rfloor, r}$. Then, it is easy to know that $\Sigma$ is invertible, and using arguments similar to (iv) of Lemma 2.1 in Chan (1990), we can conclude that for every $(\pi, \lambda) \in\left[0, \pi_{1}\right] \times\left[\lambda_{1}, \lambda_{2}\right], M_{\pi \lambda}$ is also invertible. By the ergodic theorem, we know that $X^{\top} X / n \rightarrow \Sigma$ a.s., as $n \rightarrow \infty$. Using arguments similar to Lemma 2 in Berkes et al. (2011), we can show that

$$
\sup _{0 \leq \pi \leq \pi_{1}} \sup _{\lambda_{1} \leq \lambda \leq \lambda_{2}}\left|\frac{X_{\pi \lambda}^{* \top} X}{n}-\Sigma_{\pi \lambda}\right|=o_{p}(1), \quad \text { and } \sup _{0 \leq \pi \leq \pi_{1}} \sup _{\lambda_{1} \leq \lambda \leq \lambda_{2}}\left|\frac{X_{\pi \lambda}^{* \top} X_{\pi \lambda}^{*}}{n}-\Sigma_{\pi \lambda}\right|=o_{p}(1) .
$$

Thus, we know that

$$
\sup _{0 \leq \pi \leq \pi_{1}} \sup _{\lambda_{1} \leq \lambda \leq \lambda_{2}}\left|\left\{\frac{X_{\pi \lambda}^{* \top} X_{\pi \lambda}^{*}}{n}-\frac{X_{\pi \lambda}^{* \top} X}{n}\left(\frac{X^{\top} X}{n}\right)^{-1} \frac{X^{\top} X_{\pi \lambda}^{*}}{n}\right\}^{-1}-M_{\pi \lambda}^{-1}\right|=o_{p}(1),
$$

and

$$
\begin{aligned}
& \sup _{0 \leq \pi \leq \pi_{1}} \sup _{\lambda_{1} \leq \lambda \leq \lambda_{2}}\left|T_{\pi \lambda}^{*}-\left(-\Sigma_{\pi \lambda} \Sigma^{-1}, I_{2}\right) \frac{1}{\sqrt{n}}\left(X, X_{\pi \lambda}^{*}\right)^{\top} u\right|= \\
& \sup _{0 \leq \pi \leq \pi_{1}} \sup _{\lambda_{1} \leq \lambda \leq \lambda_{2}}\left|T_{\pi \lambda}^{*}+\frac{1}{\sqrt{n}} \Sigma_{\pi \lambda} \Sigma^{-1} X^{\top} u-\frac{1}{\sqrt{n}} X_{\pi \lambda}^{* \top} u\right|=o_{p}(1),
\end{aligned}
$$

where $I_{2}$ is an identity matrix with order 2. Now, applying Theorem 8 in Li et al. (2016), under $H_{0}$, we know that $\left\{T_{\pi \lambda}^{*}\right\}$ converges weakly to $\left\{\sigma \xi_{\pi \lambda}\right\}$ in $\mathbb{D}([0,1] \times[-\infty, \infty])$, where $\mathbb{D}(A)$ is the space of real-valued functions on the set $A$, which are right continuous and have left-hand limits and it is equipped with the Skorohod topology. Using arguments similar to Theorem 1 in Zhu and Ling (2012), we know that $\hat{\sigma}_{n}^{2}$ converges to $\sigma^{2}$ a.s.,

$$
\sup _{0 \leq k \leq k_{1}} \sup _{r_{1} \leq r \leq r_{2}} S_{n}(k, r) \stackrel{d}{\longrightarrow} \sigma^{2} \sup _{0 \leq \pi \leq \pi_{1}} \sup _{\lambda_{1} \leq \lambda \leq \lambda_{2}} \xi_{\pi \lambda}^{\top} M_{\pi \lambda}^{-1} \xi_{\pi \lambda} \text { and } \frac{1}{2 n} \sup _{0 \leq k \leq k_{1}} \sup _{r_{1} \leq r \leq r_{2}} \frac{S_{n}^{2}(k, r)}{\xi_{n}^{2}(k, r)}=o_{p}(1) .
$$


From the previous arguments, it concludes that this theorem holds.

Proof of Theorem 2: First we focus on $S_{n}(k, r)=n\left(\hat{\sigma}_{n}^{2}-\hat{\sigma}_{n}^{2}(k, r)\right)$. As shown in (A.1), we have $S_{n}(k, r)=T_{k r}^{\top} R_{k r}^{-1} T_{k r}$ with

$$
\begin{aligned}
& T_{k r}=D_{n} X_{k r}^{\top} Y-D_{n} X_{k r}^{\top} X D_{n}\left(D_{n} X^{\top} X D_{n}\right)^{-1} D_{n} X^{\top} Y, \\
& R_{k r}=D_{n} X_{k r}^{\top} X_{k r} D_{n}-D_{n} X_{k r}^{\top} X D_{n}\left(D_{n} X^{\top} X D_{n}\right)^{-1} D_{n} X^{\top} X_{k r} D_{n},
\end{aligned}
$$

where $D_{n}=\operatorname{diag}\{1 / \sqrt{n}, 1 / n\}$. If $H_{0}$ holds, we have

$$
T_{k r}=D_{n} X_{k r}^{\top} u-D_{n} X_{k r}^{\top} X D_{n}\left(D_{n} X^{\top} X D_{n}\right)^{-1} D_{n} X^{\top} u
$$

Re-scale the time axis by setting $k=\lfloor n \pi\rfloor$ with $\pi \in[0,1]$, and note that $\lambda=F(r)$. Following Assumption 4 and Theorem 3 in Caner and Hansen (2001), we have

$$
\begin{aligned}
& D_{n} X_{k r}^{\top} X_{k r} D_{n}=D_{n} X_{k r}^{\top} X D_{n}=\left(\begin{array}{cc}
\frac{1}{n} \sum_{t=k+1}^{n} I_{t-1}^{*} & \frac{1}{n^{3 / 2}} \sum_{t=k+1}^{n} I_{t-1}^{*} x_{t-1} \\
\frac{1}{n^{3 / 2}} \sum_{t=k+1}^{n} I_{t-1}^{*} x_{t-1} & \frac{1}{n^{2}} \sum_{t=k+1}^{n} I_{t-1}^{*} x_{t-1}^{2}
\end{array}\right) \\
& =\left(\begin{array}{cc}
\frac{1}{n} \sum_{t=1}^{n} I_{t-1}^{*} & \frac{1}{n^{3 / 2}} \sum_{t=1}^{n} I_{t-1}^{*} x_{t-1} \\
\frac{1}{n^{3 / 2}} \sum_{t=1}^{n} I_{t-1}^{*} x_{t-1} & \frac{1}{n^{2}} \sum_{t=1}^{n} I_{t-1}^{*} x_{t-1}^{2}
\end{array}\right)-\left(\begin{array}{cc}
\frac{1}{n} \sum_{t=1}^{k} I_{t-1}^{*} & \frac{1}{n^{3 / 2}} \sum_{t=1}^{k} I_{t-1}^{*} x_{t-1} \\
\frac{1}{n^{3 / 2}} \sum_{t=1}^{k} I_{t-1}^{*} x_{t-1} & \frac{1}{n^{2}} \sum_{t=1}^{k} I_{t-1}^{*} x_{t-1}^{2} \\
\pi & \int_{0}^{\pi} B_{v}(s) d s \\
1 & \int_{0}^{1} B_{v}(s) d s \\
\left(\begin{array}{cc}
\int_{0}^{1} B_{v}(s) d s & \int_{0}^{1} B_{v}^{2}(s) d s
\end{array}\right)-\lambda\left(\begin{array}{cc}
(1-\pi) & \int_{\pi}^{1} B_{v}(s) d s \\
\int_{0}^{\pi} B_{v}(s) d s & \int_{0}^{\pi} B_{v}^{2}(s) d s
\end{array}\right) \equiv \lambda \int_{\pi}^{1} \bar{B}_{v}(s) \bar{B}_{v}^{\top}(s) d s \equiv \lambda \Omega_{\pi},
\end{array}\right. \\
& =\lambda\left(\begin{array}{cc}
\int_{\pi}^{1} B_{v}(s) d s & \int_{\pi}^{1} B_{v}^{2}(s) d s
\end{array}\right)
\end{aligned}
$$

where $\bar{B}_{v}(s)=\left(1, B_{v}(s)\right)^{\top}$. Theorem 3 in Caner and Hansen (2001) directly implies that

$$
\begin{aligned}
D_{n} X^{\top} X D_{n} & =\left(\begin{array}{cc}
1 & \frac{1}{n^{3 / 2}} \sum_{t=1}^{n} x_{t-1} \\
\frac{1}{n^{3 / 2}} \sum_{t=1}^{n} x_{t-1} & \frac{1}{n^{2}} \sum_{t=1}^{n} x_{t-1}^{2}
\end{array}\right) \Longrightarrow\left(\begin{array}{cc}
1 & \int_{0}^{1} B_{v}(s) d s \\
\int_{0}^{1} B_{v}(s) d s & \int_{0}^{1} B_{v}^{2}(s) d s
\end{array}\right) \\
& =\int_{0}^{1} \bar{B}_{v}(s) \bar{B}_{v}^{\top}(s) d s=\Omega_{0} .
\end{aligned}
$$


It now follows from the continuous mapping theorem that $R_{k r} \Longrightarrow N_{\pi \lambda}$. Using Theorems 1 and 2 in Caner and Hansen (2001), we have

$$
\begin{aligned}
D_{n} X_{k r}^{\top} u & =\left(\begin{array}{c}
\frac{1}{\sqrt{n}} \sum_{t=k+1}^{n} I_{t-1}^{*} u_{t} \\
\frac{1}{n} \sum_{t=k+1}^{n} I_{t-1}^{*} x_{t-1} u_{t}
\end{array}\right)=\left(\begin{array}{c}
\frac{1}{\sqrt{n}} \sum_{t=1}^{n} I_{t-1}^{*} u_{t} \\
\frac{1}{n} \sum_{t=1}^{n} I_{t-1}^{*} x_{t-1} u_{t}
\end{array}\right)-\left(\begin{array}{c}
\frac{1}{\sqrt{n}} \sum_{t=1}^{k} I_{t-1}^{*} u_{t} \\
\frac{1}{n} \sum_{t=1}^{k} I_{t-1}^{*} x_{t-1} u_{t}
\end{array}\right) \\
& \Longrightarrow \sigma\left(\begin{array}{c}
W(1, \lambda) \\
\int_{0}^{1} B_{v}(s) d W(s, \lambda)
\end{array}\right)-\sigma\left(\begin{array}{c}
\int_{0}^{\pi} B_{v}(s) d W(s, \lambda)
\end{array}\right) \\
& =\left(\begin{array}{c}
W(1-\pi, \lambda) \\
\int_{\pi}^{1} B_{v}(s) d W(s, \lambda)
\end{array}\right)=\sigma \int_{\pi}^{1} \bar{B}_{v}(s) d W(s, \lambda), \\
D_{n} X^{\top} u & =\left(\begin{array}{c}
\frac{1}{\sqrt{n}} \sum_{t=1}^{n} u_{t} \\
\frac{1}{n} \sum_{t=1}^{n} x_{t-1} u_{t}
\end{array}\right) \Longrightarrow \sigma\left(\begin{array}{c}
\int_{0}^{1} B_{v}(s) d W(s, 1)
\end{array}\right)=\sigma \int_{0}^{1} \bar{B}_{v}(s) d W(s, 1) .
\end{aligned}
$$

Thus, we have $T_{k r} \Longrightarrow \sigma \eta_{\pi \lambda}$. This establishes that $S_{n}(k, r) \Longrightarrow \sigma^{2} \eta_{\pi \lambda}^{\top} N_{\pi \lambda}^{-1} \eta_{\pi \lambda}$. It follows from that

$$
\hat{\sigma}_{n}^{2}=\frac{1}{n} u^{\top} u-\frac{1}{n} u^{\top} X\left(X^{\top} X\right)^{-1} X^{\top} u=\frac{1}{n} u^{\top} u-\frac{1}{n}\left\{u^{\top} X D_{n}\left(D_{n} X^{\top} X D_{n}\right)^{-1} D_{n} X^{\top} u\right\} .
$$

Using a similar argument as for the weak convergence of $T_{k r}$, we know that the second term in (A.2) converges to 0 . Furthermore, since $u^{\top} u / n$ to $\sigma^{2}$ a.s., we know that $\hat{\sigma}_{n}^{2}$ converges to $\sigma^{2}$ a.s.. Note that $\hat{\sigma}_{n}^{2}(k, r)=\hat{\sigma}_{n}^{2}-S_{n}(k, r) / n$ and $S_{n}(k, r)$ weakly converges to a limit. We know that $\hat{\sigma}_{n}^{2}(k, r)$ converges to $\sigma^{2}$ a.s. The theorem follows by standard manipulations.

Proof of Theorem 3: The argument is similar to that for Theorem 2. Note that $S_{n}(k, r)=$ $n\left(\hat{\sigma}_{n}^{2}-\hat{\sigma}_{n}^{2}(k, r)\right)=T_{k r}^{\top} R_{k r}^{-1} T_{k r}$ with

$$
\begin{aligned}
& T_{k r}=D_{n} X_{k r}^{\top} u-D_{n} X_{k r}^{\top} X D_{n}\left(D_{n} X^{\top} X D_{n}\right)^{-1} D_{n} X^{\top} u \\
& R_{k r}=D_{n} X_{k r}^{\top} X_{k r} D_{n}-D_{n} X_{k r}^{\top} X D_{n}\left(D_{n} X^{\top} X D_{n}\right)^{-1} D_{n} X^{\top} X_{k r} D_{n},
\end{aligned}
$$

where $D_{n}=\operatorname{diag}\{1 / \sqrt{n}, 1 / n\}$. Re-scale the time axis by setting $k=\lfloor n \pi\rfloor$ with $\pi \in[0,1]$, and note that $\lambda=F(r)$. Following Assumption 4 and Lemma 1 in Gonzalo and Pitarakis (2012), we have

$$
D_{n} X_{k r}^{\top} X_{k r} D_{n}=D_{n} X_{k r}^{\top} X D_{n} \Longrightarrow \lambda\left(\begin{array}{cc}
(1-\pi) & \int_{\pi}^{1} K_{c}(s) d s \\
\int_{\pi}^{1} K_{c}(s) d s & \int_{\pi}^{1} K_{c}^{2}(s) d s
\end{array}\right)
$$




$$
\equiv \lambda \int_{\pi}^{1} \bar{K}_{c}(s) \bar{K}_{c}^{\top}(s) d s \equiv \lambda \Xi_{\pi}
$$

where $\bar{K}_{c}(s)=\left(1, K_{c}(s)\right)^{\top}$. Similarly, we have

$$
D_{n} X^{\top} X D_{n} \Longrightarrow \int_{0}^{1} \bar{K}_{c}(s) \bar{K}_{c}^{\top}(s) d s=\Xi_{0} \text {. }
$$

It now follows from the continuous mapping theorem that $R_{k r} \Longrightarrow L_{\pi \lambda}$. Using Lemma 1 in Gonzalo and Pitarakis (2012), we have

$$
D_{n} X_{k r}^{\top} u \Longrightarrow \sigma\left(\begin{array}{c}
W(1-\pi, \lambda) \\
\int_{\pi}^{1} K_{c}(s) d W(s, \lambda)
\end{array}\right)=\sigma \int_{\pi}^{1} \bar{K}_{c}(s) d W(s, \lambda),
$$

and

$$
D_{n} X^{\top} u \Longrightarrow \sigma\left(\begin{array}{c}
W(1,1) \\
\int_{0}^{1} K_{c}(s) d W(s, 1)
\end{array}\right)=\sigma \int_{0}^{1} \bar{K}_{c}(s) d W(s, 1) .
$$

Thus, we have $T_{k r} \Longrightarrow \sigma \zeta_{\pi \lambda}$. This establishes that $S_{n}(k, r) \Longrightarrow \sigma^{2} \zeta_{\pi \lambda}^{\top} L_{\pi \lambda}^{-1} \zeta_{\pi \lambda}$. Using a similar argument as in (A.2), we can show that $\hat{\sigma}_{n}^{2}(k, r)$ converges to $\sigma^{2}$ a.s.. Thus, the conclusion holds by standard manipulations.

Proof of Theorem 4: We use the method as the proof of Theorem 2 in Hansen (1996), also see Theorem 3 in Zhu et al. (2014b). Re-scale the time axis and denote $z_{t}^{*}(\pi, \lambda)=z_{t}(k, r), \theta_{n}^{*}(\pi, \lambda)=$ $\theta_{n}(k, r)$ and $\hat{Z}_{n}^{*}(\pi, \lambda)=\frac{1}{\sqrt{n}} \sum_{t=1}^{n} z_{t}^{*}(\pi, \lambda) \hat{u}_{t} \varepsilon_{t}$. First, let $W$ denote the set of samples $\omega$ for which

$$
\lim _{n \rightarrow \infty} \frac{1}{n} \sum_{t=1}^{n} \sup _{\pi, \lambda}\left\|z_{t}^{*}(\pi, \lambda)\right\| u_{t}^{2}<\infty
$$

and

$$
\lim _{n \rightarrow \infty} \sup _{\pi, \lambda, \pi^{*}, \lambda^{*}}\left\|\frac{1}{n} \sum_{t=1}^{n} z_{t}^{*}(\pi, \lambda) z_{t}^{* \top}\left(\pi^{*}, \lambda^{*}\right) u_{t}^{2}-\sigma^{2} E\left(z_{t}^{*}(\pi, \lambda) z_{t}^{* \top}\left(\pi^{*}, \lambda^{*}\right)\right)\right\| \rightarrow 0 \text { a.s. }
$$

Since $\sup _{\pi, \lambda}\left\|z_{t}^{*}(\pi, \lambda)\right\| \leq \sqrt{2}\left\|X_{t}\right\|$ and $E\left(\left\|X_{t}\right\| u_{t}^{2}\right)<\infty$ due to Assumption 2, by the ergodic theorem we have

$$
\lim _{n \rightarrow \infty} \frac{1}{n} \sum_{t=1}^{n} \sup _{\pi, \lambda}\left\|z_{t}^{*}(\pi, \lambda)\right\| u_{t}^{2} \leq \lim _{n \rightarrow \infty} \frac{\sqrt{2}}{n} \sum_{t=1}^{n}\left\|X_{t}\right\| u_{t}^{2}<\infty \text { a.s }
$$

i.e., (A.3) holds. By Assumptions 1-2 and similar arguments for Theorem 1, it is not hard to see that (A.4) holds. Thus, $P(W)=1$. Take any $\omega \in W$. For the remainder of the proof, all operations are conditionally on $\omega$, and hence all of the randomness appears in the i.i.d. $N(0,1)$ variables $\left\{\varepsilon_{t}\right\}$. 
Second, define

$$
Z_{n}^{*}(\pi, \lambda)=\frac{1}{\sqrt{n}} \sum_{t=1}^{n} z_{t}^{*}(\pi, \lambda) u_{t} \varepsilon_{t}
$$

Then, using the same argument for Theorem 8 in Li et al. (2016), we have $Z_{n}(\pi, \lambda) \Longrightarrow \sigma G_{\pi \lambda}$ a.s. as $n \rightarrow \infty$, where $G_{\pi \lambda}$ is a Gaussian process with zero mean function and covariance kernel $E\left(z_{t}^{*}(\pi, \lambda) z_{t}^{* \top}\left(\pi^{*}, \lambda^{*}\right)\right)$. Note that

$$
\sup _{\pi, \lambda}\left\|\hat{Z}_{n}^{*}(\pi, \lambda)-Z_{n}^{*}(\pi, \lambda)\right\| \leq \sup _{\pi, \lambda}\left\|\frac{1}{n} \sum_{t=1}^{n} z_{t}^{*}(\pi, \lambda) z_{t}^{* \top}(\pi, \lambda) \varepsilon_{t}\right\| \sup _{\pi, \lambda}\left\|\sqrt{n}\left(\theta_{n}^{*}(\pi, \lambda)-\theta^{0}\right)\right\|,
$$

where $\theta^{0}$ is the true value of $\theta$. Using the same $\operatorname{argument}$ for $Z_{n}^{*}(\pi, \lambda)$, we have

$$
\frac{1}{n} \sum_{t=1}^{n} z_{t}^{*}(\pi, \lambda) z_{t}^{* \top}(\pi, \lambda) \varepsilon_{t} \Longrightarrow 0 \text { a.s. as } n \rightarrow \infty \text {. }
$$

Using arguments similar to Lemma A.6 in Zhu et al. (2014b), we can show that $\sup _{\pi, \lambda} \| \sqrt{n}\left(\theta_{n}^{*}(\pi, \lambda)-\right.$ $\left.\theta^{0}\right) \|=O_{p}(1)$, then, it follows that $\hat{Z}_{n}^{*}(\pi, \lambda)-Z_{n}^{*}(\pi, \lambda) \Longrightarrow 0$ in probability as $n \rightarrow \infty$. Thus, we have $\hat{Z}_{n}^{*}(\pi, \lambda) \Longrightarrow \sigma G_{\pi \lambda}$ in probability as $n \rightarrow \infty$.

Next, we consider the functional

$$
L: z(\cdot, \cdot) \in \mathbb{D}\left(\left[0, \pi_{1}\right] \times\left[\lambda_{1}, \lambda_{2}\right]\right) \rightarrow \frac{1}{\sigma^{2}} \sup _{0 \leq \pi \leq \pi_{1}} \sup _{\lambda_{1} \leq \lambda \leq \lambda_{2}} z(\pi, \lambda)^{\top} K_{\pi \lambda} z(\pi, \lambda)
$$

where $K_{\pi \lambda}=\left(-\Sigma_{\pi \lambda} \Sigma^{-1}, I_{2}\right)^{\top} M_{\pi \lambda}^{-1}\left(-\Sigma_{\pi \lambda} \Sigma^{-1}, I_{2}\right)$. Clearly, $L(\cdot)$ is a continuous functional. By the continuous mapping theory, it follows that $L\left(\hat{Z}_{n}^{*}(\pi, \lambda)\right) \Longrightarrow L\left(\sigma G_{\pi \lambda}\right)$ in probability as $n \rightarrow \infty$. Using the facts that $\hat{\sigma}_{n}^{2}$ converges to $\sigma^{2}$ a.s. and

$$
\left(-\frac{X_{k r}^{\top} X}{n}\left(\frac{X^{\top} X}{n}\right)^{-1}, I_{2}\right)^{\top}\left\{\frac{X_{k r}^{\top} X_{k r}}{n}-\frac{X_{k r}^{\top} X}{n}\left(\frac{X^{\top} X}{n}\right)^{-1} \frac{X^{\top} X_{k r}}{n}\right\}^{-1}\left(-\frac{X_{k r}^{\top} X}{n}\left(\frac{X^{\top} X}{n}\right)^{-1}, I_{2}\right)
$$

converges to $K_{\pi \lambda}$ uniformly in $(\pi, \lambda)$, we have that

$$
\sup _{0 \leq \pi \leq \pi_{1}} \sup _{\lambda_{1} \leq \lambda \leq \lambda_{2}} G \hat{L} R_{n}^{*}(\pi, \lambda)=L\left(\hat{Z}_{n}^{*}(\pi, \lambda)\right)+o_{p}(1)
$$

Based on above discussions, we have

$$
G \hat{L} R_{n} \mid w_{1}^{*}, \ldots, w_{n}^{*} \stackrel{d}{\longrightarrow} \sup _{0 \leq \pi \leq \pi_{1}} \sup _{\lambda_{1} \leq \lambda \leq \lambda_{2}} G_{\pi \lambda}^{\top} K_{\pi \lambda}^{-1} G_{\pi \lambda}
$$

in probability as $n \rightarrow \infty$. Note that the covariance kernel of $\left(-\Sigma_{\pi \lambda} \Sigma^{-1}, I_{2}\right) G_{\pi \lambda}$ is

$$
\left(-\Sigma_{\pi \lambda} \Sigma^{-1}, I_{2}\right) E\left(z_{t}^{*}(\pi, \lambda) z_{t}^{* \top}\left(\pi^{*}, \lambda^{*}\right)\right)\left(-\Sigma_{\pi^{*} \lambda^{*}} \Sigma^{-1}, I_{2}\right)^{\top}
$$




$$
\begin{aligned}
& =\left(-\Sigma_{\pi \lambda} \Sigma^{-1}, I_{2}\right)\left(\begin{array}{cc}
\Sigma & \Sigma_{\pi^{*} \lambda^{*}} \\
\Sigma_{\pi \lambda} & \Sigma_{\max \left(\pi, \pi^{*}\right), \min \left(\lambda, \lambda^{*}\right)}
\end{array}\right)\left(\begin{array}{c}
-\Sigma^{-1} \Sigma_{\pi^{*} \lambda^{*}} \\
I_{2}
\end{array}\right) \\
& =\Sigma_{\max \left(\pi, \pi^{*}\right), \min \left(\lambda, \lambda^{*}\right)}-\Sigma_{\pi \lambda} \Sigma^{-1} \Sigma_{\pi^{*} \lambda^{*}} .
\end{aligned}
$$

Thus, the conclusion holds.

The following lemma is a special case of Theorem 3 in Georgiev et al. (2019), which is used for proving Theorem 5 and stated here for convenience.

Lemma 1 Let $\tilde{e}_{n t}(t=1, \ldots, n)$ be scalar measurable functions of $y_{i}, x_{i}, q_{i}(i=1, \ldots, n)$ and such that $\frac{1}{n} \sum_{t=1}^{[n r]} \tilde{e}_{n t}^{2} \stackrel{P}{\longrightarrow} \int_{0}^{r} m^{2}(s) d s$ for $r \in[0,1]$, where $m(\cdot)$ is a square-integrable real function on $[0,1]$. Introduce $\varepsilon_{t b}=\tilde{e}_{n t} \varepsilon_{t}(t=1, \ldots, n)$, and $B^{*}(r)=\int_{0}^{r} m(s) d B_{1}^{*}(s)$, where $B_{1}^{*}$ is a standard Brownian motion independent of $\left(B_{u}, B_{v}\right)$. Under Assumption 6, the following converge jointly as

$$
\left(\frac{1}{\sqrt{n}} \sum_{t=1}^{[n r]} u_{t}, \frac{1}{\sqrt{n}} \sum_{t=1}^{[n r]} v_{t}, \frac{1}{n} \sum_{t=1}^{n} \sum_{s=1}^{t-1} v_{s} u_{t}\right) \stackrel{w}{\longrightarrow}\left(B_{u}(r), B_{v}(r), \int_{0}^{1} B_{u}(s) d B_{v}(s)\right) \mid B_{v},
$$

$r \in[0,1]$, in the sense of weak convergence of random measures on $\mathcal{D}^{2} \times \mathbb{R}$, and

$$
\left(\frac{1}{\sqrt{n}} \sum_{t=1}^{[n r]} v_{t}, \frac{1}{\sqrt{n}} \sum_{t=1}^{[n r]} \varepsilon_{t b}, \frac{1}{n} \sum_{t=1}^{n} \sum_{s=1}^{t-1} v_{s} \varepsilon_{t b}\right) \stackrel{w}{\longrightarrow}\left(B_{v}(r), B^{*}(r), \int_{0}^{1} B_{v}(s) d B^{*}(s)\right) \mid B_{v}
$$

for $r \in[0,1]$, in the sense of weak convergence of random measures on $\mathcal{D}^{2} \times \mathbb{R}$, where $\mathcal{D}^{k}:=D_{k}[0,1]$ is the space of right continuous with left limit functions from $[0,1]$ to $\mathbb{R}^{k}$, equipped with the Skorokhod topology, and $\mathcal{D}:=\mathcal{D}^{1}$.

Proof of Theorem 5: First we focus on $\hat{S}_{n}(k, r)=T_{k r}^{\star \top} R_{k r}^{-1} T_{k r}^{\star}$. Re-scale the time axis by setting $k=\lfloor n \pi\rfloor$ with $\pi \in[0,1]$, and note that $\lambda=F(r)$. Since $n^{-3 / 2} \sum_{t=1}^{n} x_{t-1} \Longrightarrow \int_{0}^{1} B_{v}^{\top}(s) d s$ by Assumption 4 and the continuous mapping theorem, we have

$$
D_{n} X_{k r}^{\top} X_{k r} D_{n}=D_{n} X_{k r}^{\top} X D_{n} \Longrightarrow \lambda \int_{\pi}^{1} \bar{B}_{v}(s) \bar{B}_{v}^{\top}(s) d s \equiv \lambda \Omega_{\pi}
$$

where $\bar{B}_{v}(s)=\left(1, B_{v}(s)\right)^{\top}$. And similarly,

$$
D_{n} X^{\top} X D_{n} \Longrightarrow \int_{0}^{1} \bar{B}_{v}(s) \bar{B}_{v}^{\top}(s) d s=\Omega_{0}
$$

It now follows from the continuous mapping theorem again that $R_{k r} \Longrightarrow N_{\pi \lambda}$. 
Next, it follows from Lemma 1 with $\tilde{e}_{n t}=\hat{u}_{t}$ that

$\left(\frac{1}{\sqrt{n}} \sum_{t=1}^{[n r]} v_{t}, \frac{1}{\sqrt{n}} \sum_{t=1}^{[n r]} I_{t-1}^{*} \hat{u}_{t}, \frac{1}{n} \sum_{t=1}^{n} \sum_{s=1}^{t-1} I_{t-1}^{*} x_{t-1} \hat{u}_{t}\right) \stackrel{w}{\longrightarrow}\left(B_{v}(r), \sigma W(r, 1), \sigma \int_{0}^{1} B_{v}(s) d W(s, 1)\right) \mid B_{v}$,

where $n^{-1} \sum_{t=1}^{[n r]} I_{t-1}^{*} \hat{u}_{t}^{2} \Longrightarrow \sigma^{2} W^{2}(r, 1)$ and $\operatorname{Var}(\sigma W(r, 1))=\sigma^{2} \operatorname{Var}(W(r, 1))=\sigma^{2} E\left(W^{2}(r, 1)\right)$ satisfying the framework of Lemma 1 . Then,

$$
\begin{aligned}
D_{n} X_{k r}^{\top} \hat{u} & =\left(\begin{array}{c}
\frac{1}{\sqrt{n}} \sum_{t=k+1}^{n} I_{t-1}^{*} \hat{u}_{t} \\
\frac{1}{n} \sum_{t=k+1}^{n} I_{t-1}^{*} x_{t-1} \hat{u}_{t}
\end{array}\right)=\left(\begin{array}{c}
\frac{1}{\sqrt{n}} \sum_{t=1}^{n} I_{t-1}^{*} \hat{u}_{t} \\
\frac{1}{n} \sum_{t=1}^{n} I_{t-1}^{*} x_{t-1} \hat{u}_{t}
\end{array}\right)-\left(\begin{array}{c}
\frac{1}{\sqrt{n}} \sum_{t=1}^{k} I_{t-1}^{*} \hat{u}_{t} \\
\frac{1}{n} \sum_{t=1}^{k} I_{t-1}^{*} x_{t-1} \hat{u}_{t}
\end{array}\right) \\
& \Longrightarrow \sigma\left(\begin{array}{c}
W(1, \lambda) \\
\int_{0}^{1} B_{v}(s) d W(s, \lambda)
\end{array}\right)\left|B_{v}-\sigma\left(\begin{array}{c}
W(\pi, \lambda) \\
\int_{0}^{\pi} B_{v}(s) d W(s, \lambda)
\end{array}\right)\right| B_{v} \\
& =\sigma\left(\begin{array}{c}
W(1-\pi, \lambda) \\
\int_{\pi}^{1} B_{v}(s) d W(s, \lambda)
\end{array}\right)\left|B_{v}=\sigma \int_{\pi}^{1} \bar{B}_{v}(s) d W(s, \lambda)\right| B_{v},
\end{aligned}
$$

and

$$
D_{n} X^{\top} \hat{u}=\left(\begin{array}{c}
\frac{1}{\sqrt{n}} \sum_{t=1}^{n} \hat{u}_{t} \\
\frac{1}{n} \sum_{t=1}^{n} x_{t-1} \hat{u}_{t}
\end{array}\right) \Longrightarrow \sigma\left(\begin{array}{c}
W(1,1) \\
\int_{0}^{1} B_{v}(s) d W(s, 1)
\end{array}\right)\left|B_{v}=\sigma \int_{0}^{1} \bar{B}_{v}(s) d W(s, 1)\right| B_{v} .
$$

Hence, $T_{k r}^{\star}\left|w_{1}^{*}, \ldots, w_{n}^{*} \Longrightarrow \sigma \eta_{\pi \lambda}\right| B_{v}$. Thus, $\hat{S}_{n}(k, r)\left|w_{1}^{*}, \ldots, w_{n}^{*} \Longrightarrow \sigma^{2} \eta_{\pi \lambda}^{\top} N_{\pi \lambda}^{-1} \eta_{\pi \lambda}\right| B_{v}$. Thus, (a) holds by standard manipulations. Finally, using Theorem 3 and a similar argument, we can show that (b) holds. 